\title{
Revisiting the pathogenic mechanism of the GJB1 5' UTR c.-103C > T mutation causing $\mathrm{CMTX} 1$
}

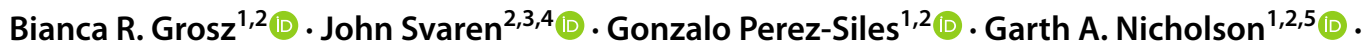 \\ Marina L. Kennerson ${ }^{1,2,5}$
}

Received: 8 March 2021 / Accepted: 25 May 2021 / Published online: 5 June 2021

(c) The Author(s) 2021

\begin{abstract}
The second most common form of Charcot-Marie-Tooth neuropathy (CMT), X-linked CMT type X1 (CMTX1), is caused by coding and non-coding mutations in the gap junction beta 1 (GJB1) gene. The non-coding $G J B 1$ c.-103C $>$ T mutation (NM_000166.5) has been reported to cause CMTX1 in multiple families. This study assessed the internal ribosomal entry site (IRES) activity previously reported for the rat Gjbl P2 5' untranslated region (UTR). Using a bicistronic assay and transfecting RT4 Schwann cells, IRES activity of the human GJB1 P2 5' UTR was compared to the GJB1 P2 5' UTR containing either the c.-103C > T mutation or the non-pathogenic c.-102G > A variant. No differences in GJB1 P2 5' UTR IRES activity were observed between the negative control, the wild-type P2 5' UTR, the c.-103C > T 5' UTR or the c.-102G $>$ A 5' UTR, irrespective of the GJB1 intron being present ( $p=.429$ with intron, and $p=.865$ without). A theoretical c. $-131 \mathrm{~A}>\mathrm{G}$ variant was predicted to result in the same RNA secondary structure as the GJB1 c.-103C > T P2 5' UTR. However, no significant difference was observed between expression from the wild-type GJB1 P2 5' UTR and the GJB1 c.-131A > G variant $(p=.688)$. Deletion of the conserved region surrounding the c.-103C $>$ T mutation (c.-108_-103del) resulted in significantly higher expression than the c.-103C $>$ T mutation alone $(p=.019)$, suggesting that the conserved c.-108_-103 region was not essential for translation. The reporter assays in this study do not recapitulate the previously reported GJB1 IRES activity and suggest an alternate pathogenic mechanism for the c.-103C > T CMTX1 non-coding mutation.
\end{abstract}

Keywords Charcot-Marie-Tooth $\cdot$ IRES $\cdot$ Neuropathy $\cdot$ Cap-independent translation $\cdot$ CMTX1

\section{Introduction}

Charcot-Marie-Tooth type X1 (CMTX1), the second most common hereditary motor and sensory peripheral neuropathy, is caused by mutations in the gap junction beta 1 (GJBI) gene. GJBI encodes the transmembrane channel protein

Bianca R. Grosz

bgro4046@uni.sydney.edu.au

1 Northcott Neuroscience Laboratory, ANZAC Research Institute, Concord, NSW, Australia

2 Sydney Medical School, University of Sydney, Camperdown, NSW, Australia

3 Waisman Center, University of Wisconsin-Madison, Madison, WI, USA

4 Department of Comparative Biosciences, University of Wisconsin-Madison, Madison, WI, USA

5 Molecular Medicine Laboratory, Concord Repatriation General Hospital, Concord, NSW, Australia connexin $32(\mathrm{Cx} 32)$ and is controlled by two alternative tissue-specific promoters (P1 and P2) that differ in the 5, untranslated region (UTR) [1]. Non-coding mutations of the neural P2 GJB1 transcript represent a significant portion of the CMTX1 cohort, with $11.4 \%$ of a UK CMTX1 cohort reporting mutations in the neural-specific GJB1 P2 promoter, 5' UTR and 3' UTR [2] (Supplementary Fig. 1). A non-coding pathogenic variant of the neural GJB1 P2 5' UTR, c.-103C> T [NM_000166.5, chrX:71,223,249 (hg38)], has been reported in a number of CMTX1 families from multiple ethnic backgrounds [3-11]. Whilst initial studies suggested the c. $-103 \mathrm{C}>\mathrm{T}$ variant completely abolished translation of Cx32 [5], a more recent luciferasebased reporter assay demonstrated the mutation decreased expression by $76.5 \%$ when co-transfected with the SOX10 transcription factor [10].

Translation commonly utilises a 5' cap-dependent mechanism in which ribosomal subunits assemble around the $5^{\prime}-\mathrm{m}_{7} \mathrm{G}$ cap end of mature mRNA. In contrast, 5' 
cap-independent translation occurs where regions of mRNA, known as an IRES, are able to recruit ribosomal subunits to initiate translation independently of the 5' cap. It was suggested that the c.-103C $>$ T mutation caused dysfunction of an IRES, as a luciferase reporter assay for the mutation showed that transcription and splicing were not affected, but the translation was abolished [5]. This study suggested that an IRES in the P2 5' UTR could allow the ribosome to bypass two upstream open reading frames (uORFs) which slow the rate of translation by causing ribosomal stalling [12]. Whilst the vast majority of viral IRES elements are well validated and supported through multiple experimental approaches [13], cellular IRES elements remain contentious and many that have been reported have not been validated using further stringent assays [14-19].

As no consensus structure or sequence exists for cellular or viral IRES elements, they must be determined experimentally. A bicistronic assay allows the direct comparison of 5' cap-dependent translation and IRES-driven 5' cap-independent translation by analysing the expression of two reporter genes from a single bicistronic mRNA. Expression of the 5' cistron relies on 5' cap-dependent translation of the bicistronic mRNA, whereas expression of the 3' cistron depends on translation initiation from an intercistronic IRES region. However, the expression of the 3' cistron may also be due to cryptic promoters or splice sites $[14,18,20]$. Determining that the intended bicistronic RNA is produced by the vector is therefore crucial when asserting IRES function [21].

Secondary structural features of the 5' UTR are not only crucial for the regulation of translation through IRES mechanisms, but also through aiding recognition of the correct translation start site and modulating the rate of translation [22]. Previous studies have demonstrated that an increase in the G-C content of RNA stem-loops close to the 5' cap results in a direct decrease in translation efficiency without affecting RNA abundance [23]. This was similar to the previously reported findings for the c. $-103 \mathrm{C}>\mathrm{T}$ mutation [5]. Given that ribosomal scanning following initiation at the 5' cap is essential for translation initiation [24, 25], it is possible that the previously reported change in the secondary structure induced by the c. $-103 \mathrm{C}>\mathrm{T}$ variant ${ }^{7}$ suggests a possible pathogenic mechanism.

The initial GJB1 P2 5' UTR experiments supporting an IRES dysfunction hypothesis were conducted using the rat Gjb1 P2 5' UTR [5], which has since been shown to differ in both sequence and secondary structure [7] when compared to human GJB1 P2 5' UTR (Supplementary Fig. 2). Additionally, the bicistronic transcript was not confirmed by RNA analysis. The effectiveness of an IRES varies amongst cell types and this is likely due to the need for cell-specific IRES trans-acting factors (ITAFs) to assist in the recruitment of the ribosome [26]. The GJB1 IRES activity was previously demonstrated by performing bicistronic assays in
HeLa cells, mouse fibroblasts (NIH-3T3) and neuroblastoma cell lines (Neuro2a) [5]. As Cx32 is expressed in Schwann cells in the peripheral nervous system, we used a Schwann cell model to reflect an appropriate tissue for CMTX1 that would likely contain the appropriate cell-specific ITAFs.

We have performed a bicistronic assay in RT4 rat Schwann cells and HeLa cells using constructs containing the human wild-type GJB1 P2 5' UTR, the pathogenic c. $-103 \mathrm{C}>\mathrm{T}$ mutation and the adjacent non-pathogenic c.$102 \mathrm{G}>\mathrm{A}$ variant [27]. Given that both sequence and structural motifs are theorised to be crucial for IRES function, additional luciferase reporter assays were developed to assess these aspects of the GJB1 P2 5' UTR. An assay was developed to assess the effect of GJB1 P2 5' UTR structure motifs on translation, as well as a deletion of the conserved sequence surrounding c. $-103 \mathrm{C}>\mathrm{T}$ to assess the functional effects of this sequence. Collectively, the results of these reporter assays do not support the role of IRES dysfunction as the pathogenic mechanism for the GJBI c. $-103 \mathrm{C}>\mathrm{T}$ mutation.

\section{Results}

The pathogenic c. $-103 \mathrm{C}>\mathrm{T}$ variant occurs in the 5' UTR of the P2 neural-specific transcript of GJB1 and causes CMTX1. A bicistronic assay was performed to reassess the previously reported IRES activity of the rat Gjbl P2 5' UTR and the effect of the pathogenic c.-103C $>$ T and non-pathogenic c.-102G $>$ A variants. Bicistronic vectors were designed in which translation of the first reporter gene (firefly luciferase; FLuc) was 5' cap-dependent, and translation of the second reporter gene (NanoLuc luciferase; NLuc) required the human GJB1 P2 5' UTR to function as an IRES and initiate translation through a 5' cap-independent mechanism (Fig. 1). The activity of FLuc acts as a control for cell viability and transfection efficiency and NLuc activity suggests IRES function of the intercistronic sequence. The known IRES from the encephalomyocarditis virus (EMCV) was used as an IRES-positive control (Fig. 1b), as this demonstrated similar IRES expression to the Gjbl P2 5' UTR previously reported [5]. Bicistronic vectors containing the full P2 5' UTR (Fig. 1c) and the P2 5' UTR with the $356 \mathrm{bp}$ GJB1 intron deletion (Fig. 1d) were used for separate transient transfections into the RT4 Schwann cell line. The bicistronic vector containing the GJB1 P2 5' UTR intron deletion (Fig. 1d) was transiently transfected into the HeLa cell line to provide a direct comparison to the previously published findings [5].

The bicistronic assay containing the $356 \mathrm{bp}$ intron (Fig. 2a) showed no significant differences in translation initiation when compared to all permutations of the GJBI P2 5' UTR bicistronic vectors (wild type, c.-103C > T, 
a

\begin{tabular}{|l|l|l|}
\hline CMV Promoter & FLuc & NLuc \\
\hline
\end{tabular}

C

i.

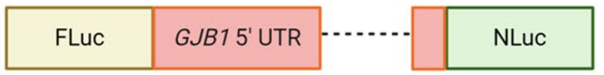

ii.

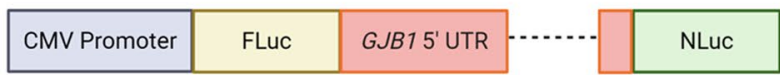

iii.

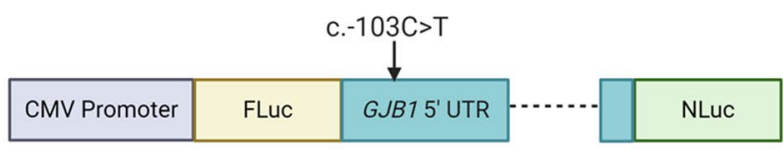

iv.

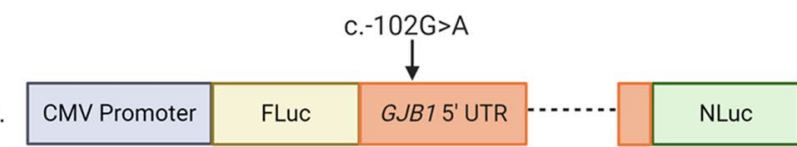

Fig. 1 GJB1 bicistronic assay design for the assessment of IRES activity. a A bicistronic vector with no intercistronic IRES serves as a negative control. b A vector containing the well-characterised viral EMCV IRES inserted between FLuc and NLuc acts as a positive control for IRES activity. c The wild-type GJB1 P2 5' UTR (ii) is inserted between the cytomegalovirus (CMV) promoter-controlled

c. $-102 \mathrm{G}>\mathrm{A})$ and negative control $(\mathrm{F}(3,8)=1.03, p=0.429)$. Similarly, the bicistronic assay results without the $356 \mathrm{bp}$ intron in RT4 cells $(\mathrm{F}(3,8)=0.242, p=0.865)$ and HeLa cells $(\mathrm{F}(3,8)=0.845, p=0.506)$ showed no significant difference when compared to the negative control (Fig. 2a). These results suggest the wild-type GJB1 P2 5' UTR does not initiate 5' cap-independent translation.

To demonstrate transcription of a complete bicistronic mRNA for the designed constructs, cDNA templates from cells transfected with each of the bicistronic constructs were analysed. Primers amplifying the transcribed full-length bicistronic mRNA showed the expected amplicon sizes for the negative IRES control (2219 bp; Fig. 2b-d), the experimental GJB1 P2 5' UTR constructs (2365 bp; Fig. 2b-d) and the positive EMCV IRES control (2788 bp; Fig. 2b-d). Sanger sequencing further confirmed the correct sequences for the different amplicons transcribed from each bicistronic vector (Fig. 2e). No amplicon was observed for the cDNA synthesis controls with no reverse transcriptase (NRTC), which confirms that the amplicons observed did not result from plasmid contamination.

Given the presence of non-pathogenic sequence variants surrounding the pathogenic c.-103C $>$ T mutation (Table 1), it was theorised that secondary RNA structures may hinder the recruitment of translation machinery. We used mFold b

\begin{tabular}{|c|c|c|c|}
\hline CMV Promoter & FLuc & EMCV IRES & NLuc \\
\hline
\end{tabular}

d

i.

\begin{tabular}{|l|l|l|l|}
\hline FLuC & GJB15' UTR & NLuc \\
\hline
\end{tabular}

ii.

ii. \begin{tabular}{|l|l|l|l|l|}
\hline CMV Promoter & FLuc & GJB15' UTR & NLuc \\
\hline
\end{tabular}
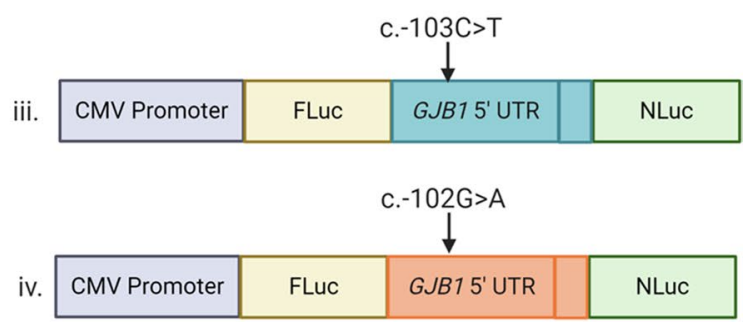

firefly luciferase (FLuc) reporter gene, and the NanoLuc luciferase (NLuc) reporter gene, as well separate GJB1 P2 5' UTR vectors harbouring the pathogenic c.-103C $>\mathrm{T}$ GJB1 (iii), and non-pathogenic c. $-102 \mathrm{G}>\mathrm{A}$ GJB1 (iv). Created with BioRender.com. d A series of experimental bicistronic assays were also created with a deletion of the $G J B 1$ intron

(version 2.4) [28] to predict secondary structural changes to the GJB1 P2 5' UTR transcript using six sequence changes (Fig. 3) wild-type GJB1 P2 5' UTR (Fig. 3a), GJB1 c.$109 \mathrm{C}>\mathrm{T}$ (Fig. 3b), GJB1 c.-103C $>$ T (Fig. 3c), GJB1 c.$102 \mathrm{G}>\mathrm{A}$ (Fig. 3d), GJB1 c.-101C $>$ T (Fig. 3e) and GJBI c. $-100 \mathrm{G}>\mathrm{A}$ (Fig. $3 \mathrm{f}$ ). The introduction of the non-pathogenic variants was predicted to lengthen a 5 ' hairpin and shortened the subsequent hairpin when compared to the wild-type secondary structure. However, the introduction of the c. $-103 \mathrm{C}>\mathrm{T}$ mutation generated a hairpin close to the 5, cap for which $10 / 13$ base pairs in the stem were stable pairings between guanine and cytosine (indicated by red lines). In contrast, for the wild-type structure, $6 / 11$ stem base pairs were G-C, and for all the other variants assessed 8/15 stem pairs were G-C. This suggested the unique RNA secondary structure caused by the GJBI c. $-103 \mathrm{C}>\mathrm{T}$ mutation may reflect a possible mechanism for pathogenicity.

To investigate if changes to the RNA secondary structure of the GJB1 P2 5' UTR caused by c.-103C > T were pathogenic, a nucleotide substitution was introduced that predicted the same GJB1 5' UTR RNA secondary structure as the c. $-103 \mathrm{C}>\mathrm{T}$ mutation. The c.-131A nucleotide forms the stem base pair opposite to GJBI c. $-103 \mathrm{C}>\mathrm{T}$, and therefore, the substitution $G J B 1$ c. $-131 \mathrm{~A}>\mathrm{G}$ was predicted by mFold to have the same RNA secondary structure as 
a

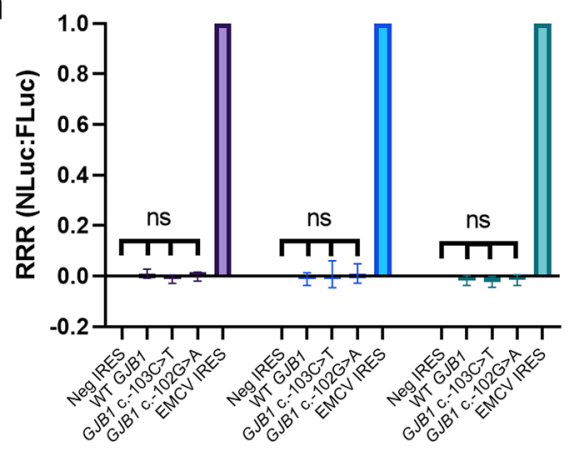

d

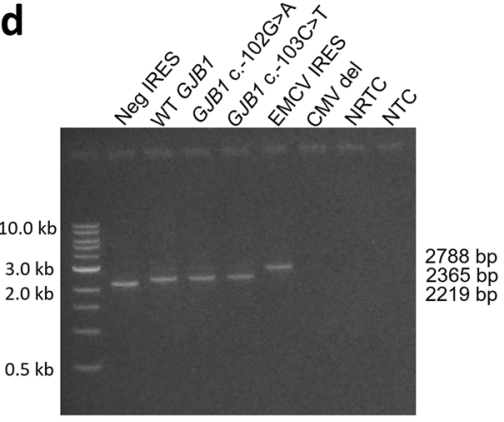

b
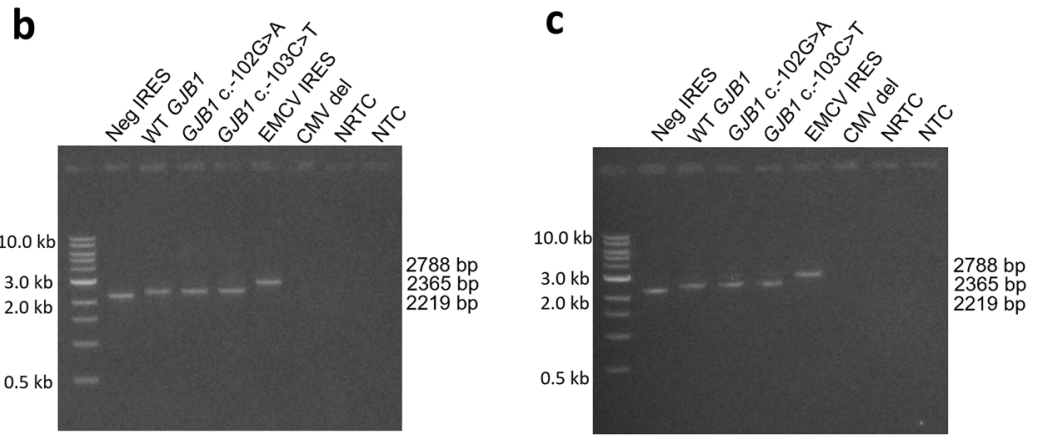

e

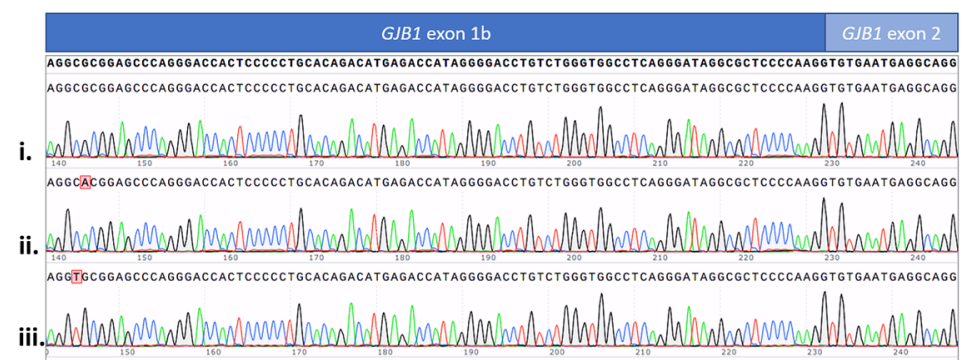

Fig. 2 The bicistronic assay to assess IRES activity of the GJB1 P2 5 ' UTR shows no evidence of increased translation of NLuc when compared to the negative IRES construct. a There was no statistically significant difference in IRES activity between the negative control, the wild-type GJB1 P2 5' UTR, the GJB1 c.-103C > T 5' UTR, GJB1 c.-102G > A 5' UTR in RT4 Schwann cells when vectors contained the GJB1 intron (purple) and without the GJB1 intron (blue). There remained no significant difference when the intronless bicistronic assay was conducted in HeLa cells (green). The relative response ratio (RRR) for each vector is shown on the y-axis, with the error bars indicating standard deviation. Statistical significance was assessed using a one-way ANOVA. Bicistronic mRNA from each vector was present for RT4 cells with GJB1 bicistronic vectors containing the

the c.-103CT > GJB1 P2 5' UTR (Fig. 4a and b). pGL4based luciferase constructs were cloned in which the FLuc gene was flanked by the GJB1 P2 promoter and 5' UTR and the GJB1 3' UTR (Fig. 4b). The different GJB1 substitutions c. $-131 \mathrm{~A}>\mathrm{G}$ (b.ii.), c. $-103 \mathrm{C}>\mathrm{T}$ (b.iii.) and nonpathogenic c.-102G $>$ A (b.iv.) were introduced into the 5', UTR. Using pRL-TK as a transfection control, the constructs were transfected separately into the RT4 cell line and FLuc and RLuc expression was measured. There was a significant intron (b), RT4 cells transfected with $G J B 1$ bicistronic vectors with the intron deleted (c) and HeLa cells transfected with GJB1 bicistronic vectors with the intron deleted $(\mathbf{d})$. A positive band is visible for the negative IRES vector (2219 bp), the three experimental GJBI P2 5' UTR vectors (2365 bp) and the positive EMCV IRES vector (2788 bp). No band was evident in the vector with the CMV promoter deletions (CMV del). NTC indicates a negative PCR reaction control (no template control). NRTC indicates a negative cDNA conversion control (no reverse transcriptase control). Size ladder (lane 1) NEB 1-kb DNA ladder. Created with BioRender.com. e Sanger sequencing confirmed correct splicing of the GJB1 P2 5' UTR for wild type (i), c. $-102 \mathrm{G}>\mathrm{A}$ (ii), and c.-103C $>$ T (iii) bicistronic mRNA

decrease in expression due to the $G J B 1$ c. $-103 \mathrm{C}>\mathrm{T}$ mutation $(M=0.16, S D=0.04)$ when compared to the wild-type GJB1 P2 5' UTR $(p<0.00001)$ (Fig. 4). However, there was no difference between the FLuc expression from the $G J B 1$ c. $-131 \mathrm{~A}>\mathrm{G}$ substitution $(M=1.12, S D=0.48)$ and wild-type $G J B 15$ ' UTR $(p=0.688)$. There was a significant difference between the FLuc expression from $G J B 1$ c.$102 \mathrm{G}>\mathrm{A}(M=0.70, S D=0.18)$ and the wild-type $G J B 1 \mathrm{P} 2$
Table 1 Reported SNPs in the GJB1 P2 5' UTR surrounding the pathogenic $G J B 1$ c. $-103 \mathrm{C}>\mathrm{T}$ variant

\begin{tabular}{lllll}
\hline SNP ID & $\begin{array}{l}\text { Coding change } \\
(\text { NM_000166.5) }\end{array}$ & Clinical significance (Clinvar) & TOPMed [44] & GnomAD [45] \\
\hline rs746618959 & c.-109C > T & Not reported & $73 / 125568$ & $5 / 21580$ \\
rs753207004 & c.-102G $>$ A & Not reported & $3 / 125568$ & $1 / 21640$ \\
rs961829342 & c.-101C > T & Likely benign (RCV000426678.1) & $15 / 125568$ & $2 / 21616$ \\
rs961626121 & c.-100G $>$ A & Not reported & $6 / 125568$ & $2 / 21621$ \\
\hline
\end{tabular}




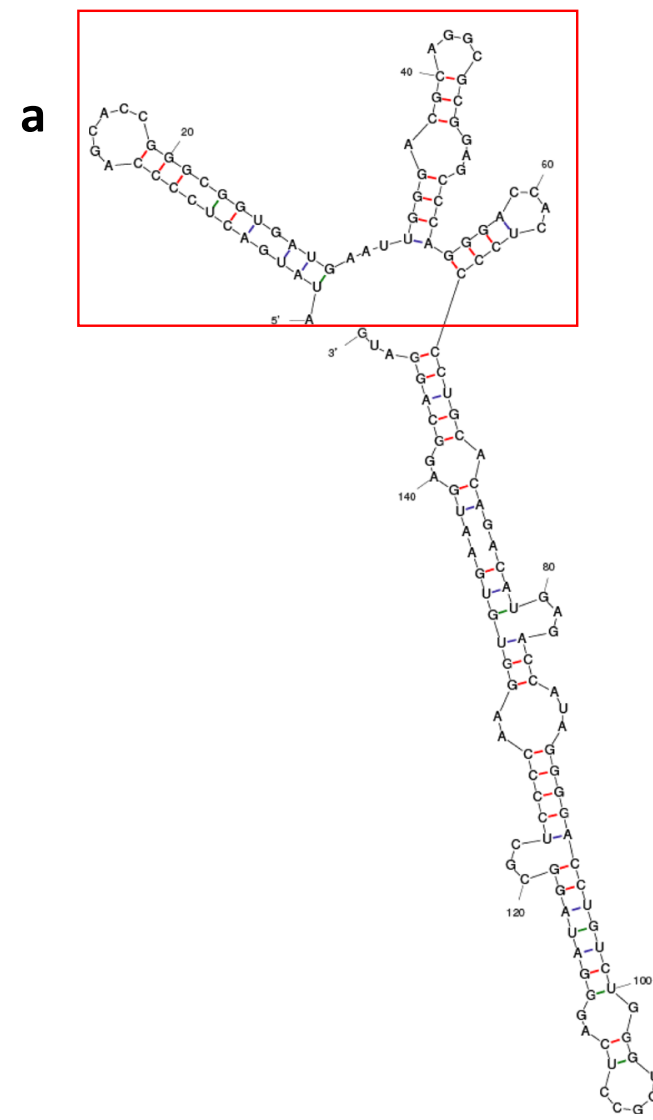

Fig. 3 Predicted secondary structures with minimum free energy for the P2 5' UTR of GJB1 spliced mRNA using mFold (version 2.4) [28] reveals that the pathogenic c.-103C $>\mathrm{T}$ mutation causes a conformational change near the 5' cap which differs to both the wild-type GJB1 P2 5' UTR and surrounding non-pathogenic variants. a Wild type. b GJB1 c. $-109 \mathrm{C}>\mathrm{T}$. c GJB1 c. $-103 \mathrm{C}>\mathrm{T}$. d GJB1c. $-102 \mathrm{G}>\mathrm{A}$. e $G J B 1$ c. $-101 C>$ T. f $G J B 1$ c. $-100 \mathrm{G}>$ A. The most thermodynami-

5' UTR ( $\mathrm{p}<0.048)$, although this variant had been reported in individuals without CMTX1.

The GJB1 c. $-103 \mathrm{C}>\mathrm{T}$ mutation occurs in an evolutionarily conserved region and is flanked by two nonpathogenic SNPs, c.-109C > T (rs746618959) and c.$102 \mathrm{G}>\mathrm{A}$ (rs753207004) (Fig. 5a). To determine if this region represents a regulatory region that is abolished by the GJB1 c. $-103 \mathrm{C}>\mathrm{T}$ mutation, an additional pGL4based luciferase deletion construct GJB1 c.-108_-103del was cloned (Fig. 5b). The GJB1 c.-108_-103del construct resulted in a $46 \%$ decrease $(M=0.54, S D=0.19)$ in expression when compared to the wild-type $G J B 1$ construct ( $\mathrm{p}=0.014)$ (Fig. 5c). However, the GJB1 c.$103 \mathrm{C}>\mathrm{T}$ mutation resulted in an $88 \%$ decrease $(M=0.12$, $S D=0.02)$ in expression when compared to the wildtype $G J B 1$ construct $(\mathrm{p}<0.00001)$. Although $G J B 1$ c.108_-103del showed a significant decrease in expression
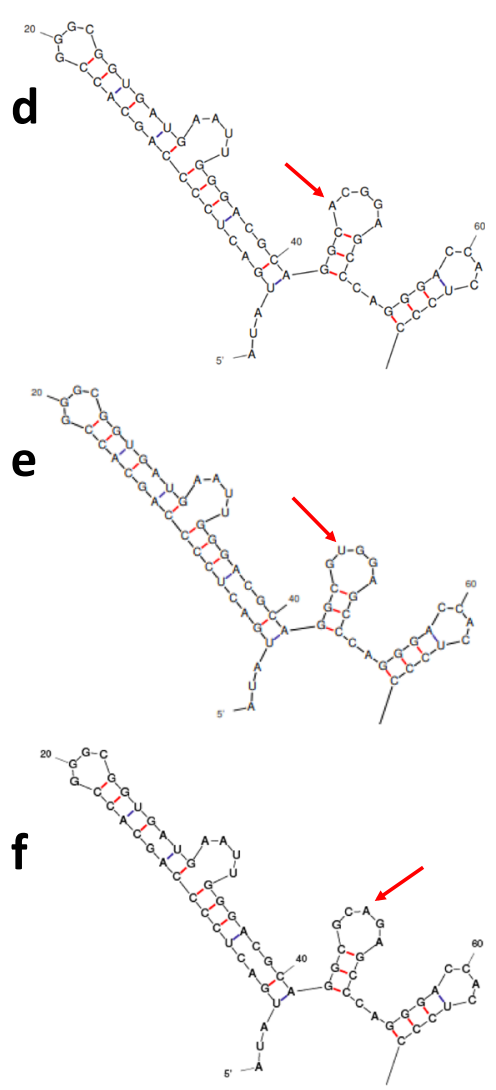

cally stable secondary structure is shown for each sequence as predicted using default parameters. The region shown for each variant is GJB1 c.-146 to c.-80 (boxed in red on the wild-type structure), as the predicted secondary structure for each variant is the same as the wild type from c.-80 to the start codon. The substituted base is indicated by an arrow

when compared to wild type, it remained significantly different from the GJB1 c. $-103 \mathrm{C}>\mathrm{T}$ mutation $(\mathrm{p}=0.019)$. This result suggests that abolishing the conserved region (c.-108 to c.-103) alone is not likely to explain the full pathogenic mechanism of the GJB1 c. $-103 \mathrm{C}>\mathrm{T}$ mutation.

\section{Discussion}

Previous studies examining the GJB1 c. $-103 \mathrm{C}>\mathrm{T}$ mutation proposed that it abolished an IRES in the GJB1 P2 5' UTR. As more IRES elements have been reported, however, the validity of many cellular IRES elements has been questioned [14-19]. The results of the bicistronic assay in our study do not support the previously proposed IRES dysfunction as a pathogenic mechanism for the GJB1 c. $-103 \mathrm{C}>\mathrm{T}$ mutation causing CMTX1. Given the 


\section{a i.}

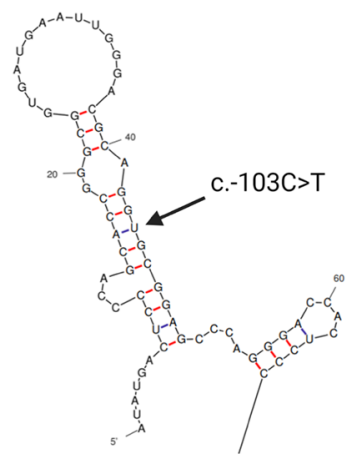

b

i.

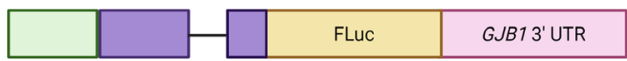

ii.

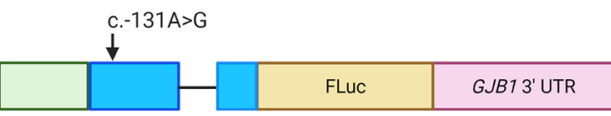

iii.

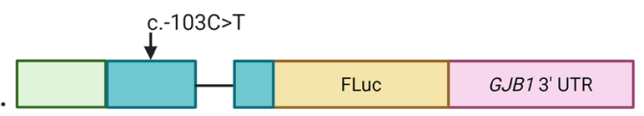

iv.

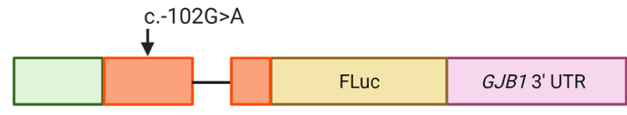

ii.

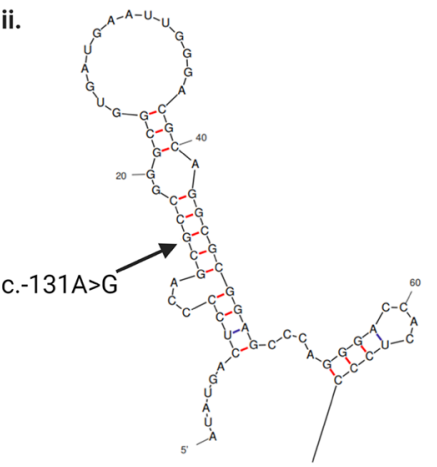

C

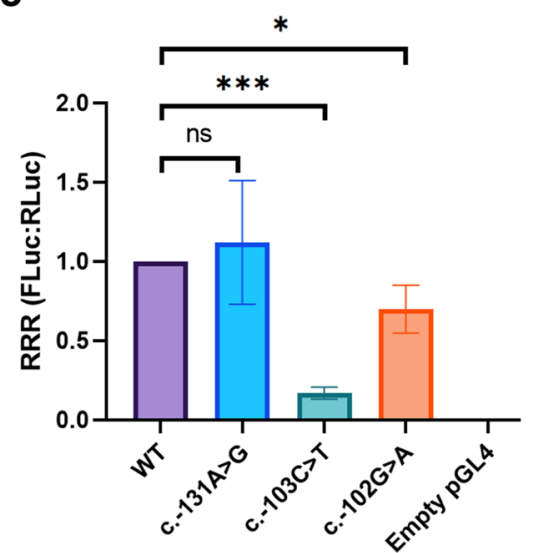

Fig. 4 GJB1 c. $-131 \mathrm{~A}>\mathrm{G}$ results in the same GJB1 5' UTR RNA secondary structure as $G J B 1$ c. $-103 \mathrm{C}>\mathrm{T}$ but does not affect expression. a The $G J B 1$ c. $-131 \mathrm{~A}>\mathrm{G}$ variant was predicted to generate the same RNA secondary structure of the GJB1 P2 5' UTR as the GJB1 c.$103 \mathrm{C}>\mathrm{T}$ mutation. b pGL4-based constructs were generated where expression of FLuc was controlled by the (i) wild-type GJB1 P2 promoter and 5' UTR (purple), as well as the GJB1 3' UTR. The (ii) GJB1 c. $-131 \mathrm{~A}>\mathrm{G}$ (blue), (iii) GJB1 c. $-103 \mathrm{C}>\mathrm{T}$ (green) and (iv) $G J B 1$ c. $-102 \mathrm{G}>\mathrm{A}$ (orange) substitutions were introduced into the

contentious nature of findings in the IRES literature, it was essential for this study to repeat the bicistronic assay using the human transcript of $G J B 1$, using the appropriate controls, and providing evidence that a full-length bicistronic mRNA was being produced in the assays. To develop effective therapeutic approaches for CMTX1 patients harbouring the GJBI c. $-103 \mathrm{C}>\mathrm{T}$ mutation, it is imperative that the underlying mechanism for the mutation could be validated and experimentally reproduced. Our results have clearly shown that abolishing an IRES element is highly unlikely to be the pathogenic mechanism.

The previously published bicistronic vectors which supported an IRES element in the GJB1 P2 5' UTR contained an initial stable stem-loop structure preceding the cap-dependent RLuc, and a second stable stem-loop structure preceding the GJB1 P2 5' UTR and cap-independent FLuc. Although not experimentally confirmed, it was suggested that the initial stem-loop would reduce high levels of cap-dependent
GJB1 5' UTR. c There was no difference between the FLuc expression from the GJB1 c.-131A > G substitution and wild-type GJB1 5, UTR $(\mathrm{p}=.688)$. There was a significant difference between the FLuc expression from the wild-type GJB1 P2 5' UTR and both GJB1 c.$102 \mathrm{G}>\mathrm{A}(\mathrm{p}<0.048)$ and $G J B 1$ c. $-103 \mathrm{C}>\mathrm{T}(\mathrm{p}<0.00001)$. The relative response ratio (RRR) for each vector is shown on the $y$-axis, with the error bars indicating standard deviation. Statistical significance was assessed using a two-tailed t-test. Created with BioRender.com

translation of RLuc, and the second stem-loop would reduce ribosomal readthrough and therefore background FLuc [5]. Comparatively, our bicistronic assay calculated the ratio of cap-dependent FLuc and cap-independent NLuc and no stable stem-loop structures were used. As the high-intensity luminescence produced by NLuc is $\sim 150$-fold greater than both FLuc and RLuc [29], an initial stable stem-loop to reduce cap-dependent FLuc expression was unnecessary. As it has been shown that IRES elements are canonically complex structures containing a series of RNA stem-loops [30, 31], we further hypothesised that a stable stem-loop preceding the GJB1 P2 5' UTR could promote false IRES activity. This was previously reported for the pim-1 5' UTR, which showed 'IRES activity' when assessed in a bicistronic vector with a series of stem-loops preceding the pim-1 5' UTR [32]. However, when the pim-1 5' UTR was stringently reassessed without these stem-loops both in vitro and in vivo, the IRES activity was no longer observed [18]. Furthermore, 
a

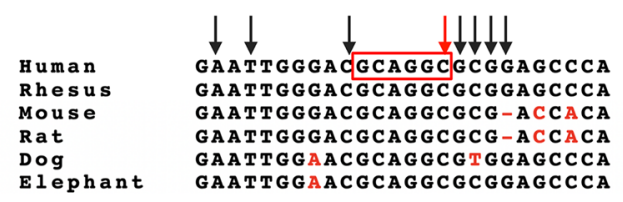

b

i.

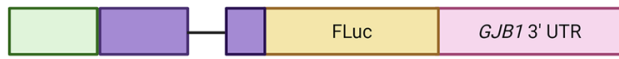

ii.

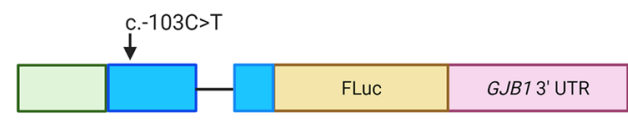

iii.

$\stackrel{1}{+}$
C

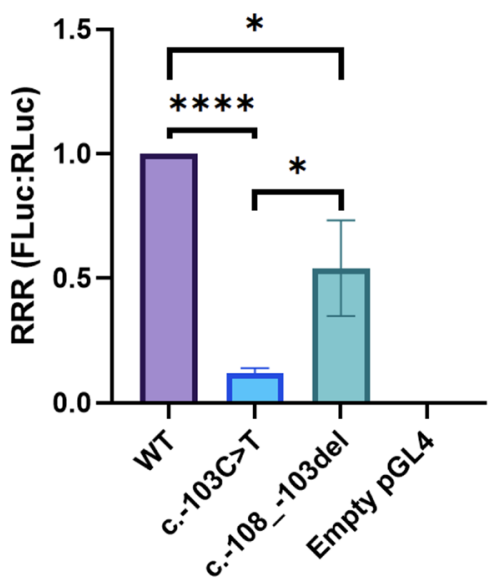

Fig. 5 The GJB1 c. $-103 \mathrm{C}>\mathrm{T}$ mutation is within an evolutionarily conserved region; however, deletion of this region did not decrease expression to GJB1 c. $-103 \mathrm{C}>\mathrm{T}$ levels. a GJB1 c. $-103 \mathrm{C}>\mathrm{T}$ (red arrow) is the last base in a 6 bp region (c.-108_-103) that is evolutionarily conserved across vertebrate species. Bases which are not conserved are highlighted in red and bases in which single-nucleotide polymorphisms have been reported in population databases are indicated by black arrows. b pGL4-based constructs were generated where expression of FLuc was controlled by the (i) wild-type GJB1 P2 promoter and 5' UTR (purple), as well as the GJB1 3' UTR. The (ii) GJB1 c. $-103 C>\mathrm{T}$ (blue) substitution was previously intro-

our modelling of the GJB1 P2 5' UTR suggested that RNA secondary structure may be crucial for its IRES function. Therefore, as suggested in the critical review of IRES validation [33], we instead utilised a series of intercistronic stop codons and ensured NLuc was out of frame with FLuc to mitigate continued ribosomal scanning of the bicistronic RNA following FLuc termination.

In this study, EMCV IRES was used as a positive control. The EMCV IRES has previously been shown to stably mediate 5' cap-independent translation in both HeLa and NIH3T3 cells [14] and was previously reported to have similar levels of IRES activity as the GJB1 P2 5' UTR IRES [5]. The bicistronic assay designed for this study used the human GJB1 P2 5' UTR in RT4 rat Schwann cells with the hypothesis that the RT4 line would contain Schwann cell-specific ITAFs and best replicate the tissue affected in CMTX1 patients. Our bicistronic assays were also conducted in HeLa cells, as this was a cell line in which the GJB1 P2 5' UTR bicistronic assay was previously conducted [5] and we were again unable to replicate the previous IRES finding.

To confirm a functional IRES, it is crucial to prove that only the intended bicistronic RNA is produced by the bicistronic vector. The initial characterisation of the GJB1 P2 5, UTR IRES did not include RNA analysis following transfection with the pRLuc-FLuc bicistronic vectors. It is therefore possible that cryptic promoter activity or unintended duced into the 5' UTR. (iii) The GJB1 c.-108_-103del (green) deletion was introduced into the GJB1 5' UTR to assess the functional effect of this region. $\mathbf{c}$ The GJB1 c.-108_-103del construct and GJB1 c. $-103 \mathrm{C}>\mathrm{T}$ both resulted significant decrease in expression when compared to the wild-type $G J B 1$ construct $(\mathrm{p}=.014$ and $\mathrm{p}<.00001$, respectively). However, the decrease in expression due to $G J B 1$ c.108_-103del was significantly different from the decrease in expression due to the $\mathrm{c} .-103 \mathrm{C}>\mathrm{T}$ mutation $(\mathrm{p}=.019)$. The relative response ratio (RRR) for each vector is shown on the $y$-axis, with the error bars indicating standard deviation. Statistical significance was assessed using a two-tailed t-test. Created with BioRender.com

splicing of the bicistronic mRNA could result in the unintended translation of the 3' cistron. Previous studies have shown that the use of a pRLuc-FLuc constructs can lead to the identification of false positive IRES elements, due to spurious splicing events caused by the presence of a chimeric intron in RLuc and strong splice donor sites in the pRLuc-FLuc vector [16, 34]. The 5' UTR of the X-linked inhibitor of apoptosis (XIAP) is an example showing purported IRES activity in a pRLuc-FLuc vector; however, cryptic promoter activity and splicing were demonstrated to mediate this apparent IRES activity [14, 17, 34, 35]. The removal of 5' splice donor sites upstream of a putative IRES in a pGluc-GFP bicistronic vector removed the apparent IRES activity of four of six reported eukaryotic IRES due to the presence of a 3' splice acceptor site within the putative IRES sequence [14]. It was previously determined that mutating a polypyrimidine tract of the GJB1 P2 5' UTR could increase its apparent IRES activity; however, polypyrimidine tracts are also known to be part of the consensus sequence that identifies a 3' splice acceptor site [36].

Studies have also suggested that the 5' UTRs of both Cx26 and $\mathrm{Cx} 43$ contain an IRES, and these experiments were conducted using the same bicistronic vector system as for $\mathrm{Cx} 32$ (pRLuc-FLuc) [37, 38]. However, the possibility of a cryptic promoter was only excluded for $\mathrm{Cx} 26$ [37]. Recent attempts to recapitulate the previously reported IRES activity of three 
isoforms of the Cx43 5' UTR were unsuccessful [39]. The Cx43 transcript has been reported to contain an internal IRES leading to translation of a smaller $20 \mathrm{kDa} \mathrm{Cx} 43$ isoform; however, the subsequent analysis determined that upstream scanning or 5' cap-dependent translation by the ribosome is necessary to initiate this internal translation [15]. It is possible that the 5' UTR of GJB1 may act in the same way, where elements of the 5' UTR are required to regulate translation following the canonical recognition of the 5' cap by the ribosome and therefore is not truly 'cap independent'.

There are several lines of evidence which do not support the role of IRES dysfunction in the pathogenicity of the GJB1 c.$103 \mathrm{C}>\mathrm{T}$ mutation in CMTX1. Initially, it was suggested that the GJB1 c.-103C > T mutation was immediately upstream of a GNRA tetraloop motif sequence ( $\mathrm{N}$ refers to any nucleotide, $\mathrm{R}$ refers to $\mathrm{G}$ or A) [5], which is a motif that has been shown to regulate the activity of viral IRES elements [40-42]. However, subsequent iterations of the human reference genome have revealed the insertion of an additional base in this region, and therefore, this motif is no longer present. Whilst deletion of the conserved region upstream of GJBI c. $-103 \mathrm{C}>\mathrm{T}$ (c.108_-103del) did decrease expression when compared to the wild-type GJB1 P2 5' UTR, the decrease was significantly different from the c.-103C $>$ T mutation. Additionally, variants in the three bases immediately downstream of the $G J B 1$ c. $-103 \mathrm{C}>\mathrm{T}$ mutation $($ GJB1 c. $-102 \mathrm{G}>\mathrm{A}$, GJBI c. $-101 \mathrm{C}>\mathrm{T}$, $G J B 1$ c. $-100 \mathrm{G}>\mathrm{A})$, as well as six bases upstream $(G J B 1$ c.$109 \mathrm{C}>\mathrm{T}$ ), represent variants present in databases at frequencies that do not support pathogenicity (Table 1). Collectively, these results do not suggest that recognition of this region is necessary for translation, and therefore, it is unlikely that this region is an IRES required for $\mathrm{Cx} 32$ translation. Instead, these results suggest that the $G J B 1$ c. $-103 \mathrm{C}>\mathrm{T}$ mutation is possibly pathogenic due to the creation of a negative sequence motif, such as a repressor binding site, rather than the disruption of a functionally important element.

Overall, the bicistronic assays designed in this study revealed no evidence of an IRES in the 5' UTR of GJB1, regardless of the presence of the GJBI intron or the cell line used. Similarly, the bicistronic vectors containing either the pathogenic (c. $103 \mathrm{C}>\mathrm{T}$ ) or non-pathogenic (c.-102G $>$ A) variant showed no IRES activity. Further luciferase reporter assays did not support aberrant RNA secondary structure or the disruption of a functional regulatory element in the GJB1 P2 5' UTR as pathogenic mechanisms for the c.-103C $>$ T mutation. Collectively, these results do not support the role of IRES dysfunction as the pathogenic mechanism for the $G J B 1$ c. $-103 \mathrm{C}>\mathrm{T}$ mutation and alternative explanations warrant consideration. Further investigation to fully define a pathogenic mechanism will enable suitable strategies for developing treatment therapies for CMTX1.

\section{Materials and methods}

\section{Standard protocol approvals, registrations and patient consents}

Individuals participating in this study were enrolled through the Neurogenetics Clinic Concord Hospital, Sydney. Genomic DNA was isolated from peripheral blood. These procedures were performed with informed consent according to protocols approved by the Sydney Local Health District, Human Ethics Committee, Concord Hospital, Australia (HREC/17/CRGH/8).

\section{Generation of bicistronic luciferase vectors}

The existing reporter vector CMV-Xbp1-FLuc2a-NLuc2aPuro [43] was modified through multiple steps using the Q5® Site-Directed Mutagenesis (SDM) Kit (New England Biolabs, Beverly, MA, USA) to generate the bicistronic vectors in Fig. 1. Transformations were performed with One Shot TOP10 chemically competent E. coli (Invitrogen, Carlsbad, CA 92,008 USA) and plasmid DNA was subsequently purified using the Isolate II Plasmid Mini Kit (Bioline). All plasmids underwent Sanger sequencing to verify the introduced mutations and variants using the BigDye Terminator Cycle Sequencing protocols at Garvan Molecular Genetics (Garvan Institute of Medical Research, Australia).

To generate the bicistronic vector pCMV-FLuc-NLuc (Fig. 1b), deletion of Xbp1 from CMV-Xbp1-FLuc2aNLuc2a-Puro was performed using Xbp1del_F and Xbp1del_R primers that flanked the Xbp1 region (Table 2). Insertion of stop codons downstream of NLuc and deletion of the $2 \mathrm{aNeo}$ region was then performed using primers that flanked the $2 \mathrm{aNeo}$ region with stop codons added to the 5' end of the forward primer, 2aNeodel_NLucStop_F and 2aNeodel_NLucStop_R (Table 2). A series of stop codons downstream of FLuc and unique HindIII and AflII restriction sites were inserted in the intercistronic region between FLuc and NLuc through the addition of half of the insertion sequence to the $5^{\prime}$ end of each primer, FLucStop_HindIIIAflII_F and FLucStop_HindIIIAflII_R (Table 2). The stop codons ensured that the ribosome would terminate translation following translation of FLuc, and additional bases ensured that FLuc and NLuc were out of frame to mitigate ribosomal readthrough leading to expression of NLuc [33]. The intercistronic 2a region was then deleted using 2adel_F and 2adel_R (Table 2). This vector served as the negative control (Fig. 1a).

A positive IRES control was generated by amplifying the encephalomyocarditis (EMCV) IRES region from 
Table 2 Primer names and sequences used for the cloning of luciferase reporter vectors

\begin{tabular}{|c|c|}
\hline Primer name & Sequence $\left(5^{\prime}-3^{\prime}\right)$ \\
\hline Xbp1del_F & TCGCCCATGGAAGATGCC \\
\hline Xbp1del_R & GCTAGCCAGCTTGGGTCT \\
\hline 2aNeodel_NLucStop_F & taataaGATCGACCGATGCCCTTG \\
\hline 2aNeodel_NLucStop_R & AGCCAGAATGCGTTCGCA \\
\hline FLucStop_HindIIIAflII_F & gcggcggaagcttGGAGAGGGCAGAGGAAGT \\
\hline FLucStop_HindIIIAflII_R & ttaagttattattaAGAATTCACGGCGATCTTG \\
\hline 2adel_F & GCTCGAGTTTTTGGCATCTTC \\
\hline 2adel_R & AAGCTTCCGCCGCTTAAG \\
\hline EMCVIRES_AflII_F & AAAAAAAAGCTTATATGACTCCCCAGCACCGG \\
\hline EMCVIRES_XhoI_R & AAAAAACTCGAGCCTGCCTCATTCACACCTGCAA \\
\hline GJB1P25UTR_HindIII_F & AAAAAAAAGCTTATATGACTCCCCAGCACCGG \\
\hline GJB1P25UTR_XhoI_R & AAAAAACTCGAGCCTGCCTCATTCACACCTGCAA \\
\hline GJB1_WTSDM_F & GGGACGCAGGcGCGGAGCCCA \\
\hline GJB1_WTSDM_R & AATTCATCACCGCCCGGTGCTGG \\
\hline GJB1_102GASDM_F & GGACGCAGGCaCGGAGCCCAG \\
\hline GJB1_102GASDM_R & CAATTCATCACCGCCCGGTGC \\
\hline GJB1_Introndel_F & GTGTGAATGAGGCAGGATGATATG \\
\hline GJB1_Introndel_R & CTTGGGGAGCGCCTATCC \\
\hline CMVPromdel_F & ATCTGGCTAGCTCGCCCATG \\
\hline CMVPromdel_R & CGATTCACACAAAAAACCAACAC \\
\hline GJB1P2Prom5UTR_HindIII_F & AAAAAACTCGAGATCCACCTGCCTGTGTTTTATCTC \\
\hline GJB1P2Prom5UTR_XhoI_R & AAAAAAAAGCTTCCTGCCTCATTCACACCTGCAA \\
\hline GJB13UTR_F & AAAAAATCTAGATGCCACATACCAGGCAAC \\
\hline GJB13UTR_R & AAAAAAGGCCGGCCTTCAGAGGGAGTTGTCATTTTTAATC \\
\hline HindIII_ATGdel_F & ATGGAAGATGCCAAAAAC \\
\hline HindIII_ATGdel_R & CCTGCCTCATTCACACCT \\
\hline GJB1_103CTSDM_F & GGGACGCAGGtGCGGAGCCCA \\
\hline GJB1_103CTSDM_R & AATTCATCACCGCCCGGTGCTGG \\
\hline GJB1_131AGSDM_F & ACTCCCCAGCgCCGGGCGGTG \\
\hline GJB1_131AGSDM_R & CATATGCTGCTTTATACCCAGTGTCTG \\
\hline GJB1_108103delSDM_F & GCGGAGCCCAGGGACCAC \\
\hline GJB1_108103delSDM_R & GTCCCAATTCATCACCGCCCG \\
\hline
\end{tabular}

Mutagenesis sequences are indicated in lower case
pLPCX-Cx43-IRES-GFP [15] using EMCVIRES_AfIII_F and EMCVIRES_XhoI_R (Table 2).pLPCX-Cx43-IRESGFP was a gift from Trond Aasen (Addgene plasmid \# 65,433; http://n2t.net/addgene:65433; RRID:Addgene $(65,433)$. The amplicon was cloned into the bicistronic pCMV-FLuc-NLuc vector in the intercistronic region between FLuc and NLuc using the AflII/XhoI sites to generate pCMV-FLuc-EMCV-NLuc (Fig. 1b).

To generate the GJB1 P2 5' UTR bicistronic vectors, pCMV-FLuc-GJB1 P2 5'UTR-NLuc, the genomic region coding for the GJB1 P2 5' UTR (chrX:71,223,206-71,223,707 hg38) was amplified from a patient with the GJB1 c. $-103 \mathrm{C}>\mathrm{T}$ mutation using GJB1P25UTR_HindIII_FGJB1P25UTR_XhoI_R (Table 2). The amplicon was cloned into the bicistronic pCMV-FLucNLuc vector in the intercistronic region between FLuc and
NLuc using the HindIII/XhoI sites. Q5 SDM was used to correct the GJB1 c.-103C > T mutation to wild type using GJB1_WTSDM_F and GJB1_WTSDM_R (Table 2) and the $G J B 1$ c. $-102 \mathrm{G}>\mathrm{A}$ polymorphism was introduced using GJB1_102GASDM_F and GJB1_102GASDM_R.

Vectors without the GJB1 intron (Fig. 1d) were also generated for the wild-type GJB1 P2 5' UTR, GJB1 c.-103C > T 5' UTR and $G J B 1$ c. $-102 \mathrm{G}>\mathrm{A}$ using primers flanking the 356 bp intron, GJB1_Introndel_F and GJB1_Introndel_R (Table 2).

To generate a promoterless control (Fig. 1c (i) and d (i)) for the assessment of background luminescence (Fig. 1a), the CMV promoter of the pCMV-FLuc-GJB1P25'UTRNLuc vectors (both with and without the intron) was deleted using primers which flank the CMV promoter region, CMVPromdel_F and CMVPromdel_R (Table 2). 


\section{Generation of pGL4-based reporter luciferase vectors}

The promoterless pGL4.10[luc2] luciferase reporter vector was used to generate a suite of reporter constructs to assess the expression of the GJB1 $\mathrm{P} 2$ promoter and 5' UTR. The GJB1 P2 promoter and 5' UTR (hg38chrX:71,222,954-71,223,707) were amplified from control genomic DNA using GJB1P2Prom5UTR_HindIII_F and GJB1P2Prom5UTR_XhoI_R (Table 2). This amplicon was inserted between the HindIII and XhoI restriction sites in the pGL4.10[luc2] vector. The GJB1 3' UTR (hg38chrX:71,224,560-71,225,516) was amplified from control genomic DNA using GJB13UTR_F and GJB13UTR_R (Table 2). This amplicon was inserted between the XbaI and FseI sites in the pGL4.10[luc2] vector with the GJB1 P2 promoter and 5' UTR previously inserted. For the GJB1 5' UTR to directly control the translation of FLuc, Q5 SDM was utilised to delete the region encompassing the HindIII recognition site to the base preceding the FLuc start codon using HindIII_ATGdel_F and HindIII_ATGdel_R (Table 2).

This vector was then used as a template for Q5 SDM to introduce the c. $-103 \mathrm{C}>\mathrm{T}$ mutation using GJB1_103CTSDM_F and GJB1_103CTSDM_R (Table 2), the c.-102G > A variant using GJB1_102GASDM_F and GJB1_102GASDM_R (Table 2), the c.-131A > G variant using GJB1_131AGSDM_F and GJB1_131AGSDM_R (Table 2) and the c.-108_-103del variant using GJB1_108103delSDM_F and GJB1_108103delSDM_R (Table 2).

\section{Cell culture}

Rat Schwann cells (RT4) were cultured in a 96-well plate in Dulbecco's Modified Eagle's Medium (DMEM: Gibco) with $10 \%$ (v/v) foetal bovine serum (Gibco) at $37{ }^{\circ} \mathrm{C}$ with $5 \%$ $\mathrm{CO}_{2}$ until they reached 70-80\% confluency. HeLa cells were cultured in a 96-well plate in DMEM (Gibco) with $10 \%$ (v/v) foetal bovine serum (Gibco) and $2 \mathrm{mM}$ L-glutamine (Gibco) at $37{ }^{\circ} \mathrm{C}$ with $5 \% \mathrm{CO}_{2}$ until they reached $80-90 \%$ confluency.

\section{Bicistronic assay transfection}

Cells were transiently transfected with $100 \mathrm{ng}$ per well of a bicistronic vector using Lipofectamine 3000 (Invitrogen) according to the manufacturer's instructions. All bicistronic assays were repeated in triplicate, with each vector transfected into four wells for each assay. Each bicistronic assay was conducted with the negative IRES control (Fig. 1a) and the positive EMCV control (Fig. 1b). The GJB1 bicistronic vectors containing the $356 \mathrm{bp} G J B 1$ intron (Fig. 1c) were tested in the RT4 cell line, and the GJB1 bicistronic vectors containing the 356 bp GJB1 intron (Fig. 1d) were tested in both the RT4 Schwann cell line and HeLa cell line.

\section{Bicistronic assay}

A dual-luciferase assay was performed $48 \mathrm{~h}$ post-transfection using the NanoGlo Dual Luciferase Assay (Promega) according to the manufacturer's instructions for 96-well plates using multichannel pipettes. FLuc and NLuc activity was measured using a luminometer (Perkin Elmer Enspire II) $15 \mathrm{~min}$ post the addition of their respective substrates. Luminescence was normalised against the appropriate promoterless vector and the ratio of NLuc:FLuc was calculated. Using these values, the relative response ratios (RRR) were calculated where the ratio of NLuc:FLuc for the positive EMCV IRES control was set to a value of 1 and the ratio of NLuc:FLuc for the negative IRES control was set to a value of 0 using the following equation:

$$
R R R=\frac{\text { experimental ratio }- \text { negative IRES ratio }}{\text { positive EMCV ratio }- \text { negative IRES ratio }}
$$

Statistical significance was assessed using a one-way ANOVA with a p-value $<0.05$ suggesting statistical significance.

\section{RNA extraction and analysis}

Cultured rat Schwann cells (RT4) were grown in a 6-well plate in Dulbecco's Modified Eagle's Medium (DMEM) with $10 \%(\mathrm{v} / \mathrm{v})$ foetal bovine serum until they reached $70-80 \%$ confluency. These cells were then transiently transfected with $2000 \mathrm{ng}$ of a bicistronic assay vector using Lipofectamine 3000 (Invitrogen) according to manufacturer's instructions. The same experimental procedure was repeated using HeLa cells, which were cultured in a 96-well plate in Dulbecco's Modified Eagle's Medium (DMEM) with 10\% (v/v) foetal bovine serum and $2 \mathrm{mM}$ L-glutamine (Gibco) at $37^{\circ} \mathrm{C}$ with $5 \% \mathrm{CO}_{2}$ until they reached $80-90 \%$ confluency. RNA was extracted from the cell lines $48 \mathrm{~h}$ post-transfection using the RNEasy Mini Kit (Qiagen). RNA was then reverse transcribed using iScript cDNA Synthesis Kit (Bio$\mathrm{Rad})$. A PCR amplification was performed using primers which amplify the complete bicistronic mRNA, which was sequenced using the BigDye Terminator Cycle Sequencing protocols at Garvan Molecular Genetics (Garvan Institute of Medical Research, Australia).

\section{pGL4-based reporter luciferase assays}

RT4 cells were cultured as described previously in 96-well TC-treated plates. Cells were transiently transfected with 
$100 \mathrm{ng}$ per well of a pGL4-based reporter vector using Lipofectamine 3000 (Invitrogen) according to the manufacturer's instructions. Ten nanograms of pRL-TK (Promega) was co-transfected and RLuc expression was used as a transfection and cell viability control. All bicistronic assays were repeated in triplicate, with each vector transfected into three wells for each assay. Each assay was conducted with the wild-type GJBI-FLuc vector as a positive control and an empty pGL4 vector as the negative control.

A dual-luciferase assay was performed $48 \mathrm{~h}$ post-transfection using the Dual-Glo® Dual Luciferase Assay (Promega) according to manufacturer's instructions for 96-well plates using multichannel pipettes. FLuc and RLuc activity was measured using a luminometer (Perkin Elmer Enspire II) $15 \mathrm{~min}$ after the addition of their respective substrates. Luminescence was normalised against the background and the ratio of FLuc:RLuc was calculated. Using these values, the relative response ratios (RRR) were calculated where the ratio of FLuc:RLuc for the wild-type GJB1-pGL4 vector was set to a value of 1 and the ratio of FLuc:RLuc for the empty pGL4 vector was set to a value of 0 using the following equation:

$$
R R R=\frac{\text { experimental ratio }- \text { empty } p G L 4 \text { ratio }}{\text { wild type GJB1 }-p G L 4 \text { ratio }- \text { empty } p G L 4 \text { ratio }}
$$

Statistical significance was assessed using a twotailed t-test with a $\mathrm{p}$-value $<0.05$ suggesting statistical significance.

Supplementary Information The online version contains supplementary material available at https://doi.org/10.1007/s10048-021-00650-9.

Author contributions All authors contributed to the study conception and design. Material preparation, data collection and analysis were performed by Bianca R. Grosz. The first draft of the manuscript was written by Bianca R. Grosz and all authors commented on previous versions of the manuscript. All authors read and approved the final manuscript.

Funding This research is supported by an Australian Government Research Training Program (RTP) Scholarship.

Availability of data and material (data transparency) Not applicable.

Code availability (software application or custom code) Not applicable.

\section{Declarations}

Ethics approval These procedures were performed with informed consent according to protocols approved by the Sydney Local Health District, Human Ethics Committee, Concord Hospital, Australia (HREC/17/CRGH/8)

Consent to participate Not applicable.

Consent for publication Not applicable.
Conflict of interest The authors declare no competing interests.

Open Access This article is licensed under a Creative Commons Attribution 4.0 International License, which permits use, sharing, adaptation, distribution and reproduction in any medium or format, as long as you give appropriate credit to the original author(s) and the source, provide a link to the Creative Commons licence, and indicate if changes were made. The images or other third party material in this article are included in the article's Creative Commons licence, unless indicated otherwise in a credit line to the material. If material is not included in the article's Creative Commons licence and your intended use is not permitted by statutory regulation or exceeds the permitted use, you will need to obtain permission directly from the copyright holder. To view a copy of this licence, visit http://creativecommons.org/licenses/by/4.0/.

\section{References}

1. Neuhaus IM, Dahl G, Werner R (1995) Use of alternate promoters for tissue-specific expression of the gene coding for connexin 32 . Gene. https://doi.org/10.1016/0378-1119(94)00899-4

2. Tomaselli PJ, Rossor AM, Horga A et al (2017) Mutations in noncoding regions in GJB1 are a major cause of X-linked CMT. Neurology 88:1445-1453. https://doi.org/10.1212/WNL.00000 00000003819

3. Ionasescu VV, Searby C, Ionasescu R et al (1996) Mutations of the noncoding region of the connexin 32 gene in X-linked dominant Charcot-Marie-Tooth neuropathy. Neurology 47:541-544. https:// doi.org/10.1212/WNL.47.2.541

4. Flagiello L, Cirigliano V, Strazzullo M, et al (1998) Mutation in the nerve-specific 5'non-coding region of $\mathrm{Cx} 32$ gene and absence of specific mRNA in a CMTX1 Italian family. Mutations in brief no. 195. Online. Hum Mutat 12:361

5. Hudder A, Werner R (2000) Analysis of a Charcot-Marie-Tooth disease mutation reveals an essential internal ribosome entry site element in the connexin-32 gene. J Biol Chem 275:34586-34591. https://doi.org/10.1074/jbc.M005199200

6. Mandich P, Grandis M, Geroldi A et al (2008) Gap junction beta 1 (GJB1) gene mutations in Italian patients with X-linked CharcotMarie-Tooth disease. J Hum Genet 53:529-533. https://doi.org/ 10.1007/s10038-008-0280-4

7. Li M, Cheng T-S, Ho PW-L et al (2009) $-459 \mathrm{C}>\mathrm{T}$ point mutation in 5' non-coding region of human GJB1 gene is linked to X-linked Charcot-Marie-Tooth neuropathy. J Peripher Nerv Syst 14:14-21. https://doi.org/10.1111/j.1529-8027.2009.00201.x

8. Kabzińska D, Kotruchow K, Ryniewicz B, Kochański A (2011) Two pathogenic mutations located within the 5'-regulatory sequence of the GJB1 gene affecting initiation of transcription and translation. Acta Biochim Pol 58:359-363

9. Shahrizaila N, Samulong S, Tey S et al (2013) X-linked CharcotMarie-Tooth disease predominates in a cohort of multiethnic Malaysian patients. Muscle Nerve 49:198-201. https://doi.org/ 10.1002/mus.23892

10. Tsai P-C, Chen C-H, Liu A-B et al (2013) Mutational analysis of the $5^{\prime}$ non-coding region of GJB1 in a Taiwanese cohort with Charcot-Marie-Tooth neuropathy. J Neurol Sci 332:51-55. https://doi.org/10.1016/j.jns.2013.06.011

11. Liu X, Duan X, Zhang Y et al (2020) Cross-sectional study in a large cohort of Chinese patients with GJB1 gene mutations. Front Neurol 11:690. https://doi.org/10.3389/fneur.2020.00690

12. Calvo SE, Pagliarini DJ, Mootha VK (2009) Upstream open reading frames cause widespread reduction of protein expression 
and are polymorphic among humans. Proc Natl Acad Sci U S A 106:7507-7512. https://doi.org/10.1073/pnas.0810916106

13. Yamamoto H, Unbehaun A, Spahn CMT (2017) Ribosomal chamber music: toward an understanding of IRES mechanisms. Trends Biochem Sci 42:655-668. https://doi.org/10.1016/J.TIBS.2017. 06.002

14. Baranick BT, Lemp NA, Nagashima J et al (2008) Splicing mediates the activity of four putative cellular internal ribosome entry sites. Proc Natl Acad Sci U S A 105:4733-4738. https://doi.org/ 10.1073/pnas.0710650105

15. Salat-Canela C, Sesé M, Peula C et al (2014) Internal translation of the connexin 43 transcript. Cell Commun Signal 12:31. https:// doi.org/10.1186/1478-811X-12-31

16. Holcik M, Graber T, Lewis SM et al (2005) Spurious splicing within the XIAP 5' UTR occurs in the Rluc/Fluc but not the betagal/CAT bicistronic reporter system. RNA 11:1605-1609. https:// doi.org/10.1261/rna.2158605

17. Bert AG, Grépin R, Vadas MA, Goodall GJ (2006) Assessing IRES activity in the HIF- $1 \alpha$ and other cellular 5' UTRs. RNA. https://doi.org/10.1261/rna.2320506

18. Wang Z, Weaver M, Magnuson NS (2005) Cryptic promoter activity in the DNA sequence corresponding to the pim-1 5'-UTR. Nucleic Acids Res. https://doi.org/10.1093/nar/gki523

19. Han B, Zhang J-T (2002) Regulation of Gene Expression by Internal Ribosome Entry Sites or Cryptic Promoters: the eIF4G Story. Mol Cell Biol 22:7372-7384. https://doi.org/10.1128/mcb.22.21. 7372-7384.2002

20. Han B, Zhang J-T (2002) Regulation of gene expression by internal ribosome entry sites or cryptic promoters: the eIF4G Story. Mol Cell Biol 22:7372-7384. https://doi.org/10.1128/mcb.22.21. $7372-7384.2002$

21. Kozak M (2005) A second look at cellular mRNA sequences said to function as internal ribosome entry sites. Nucleic Acids Res 33:6593-6602. https://doi.org/10.1093/nar/gki958

22. Gingras A-C, Raught B, Sonenberg N (1999) eIF4 Initiation factors: effectors of mRNA recruitment to ribosomes and regulators of translation. Annu Rev Biochem 68:913-963. https://doi.org/10. 1146/annurev.biochem.68.1.913

23. Babendure JR, Babendure JL, Ding JH, Tsien RY (2006) Control of mammalian translation by mRNA structure near caps. RNA 12:851-861. https://doi.org/10.1261/rna.2309906

24. Kozak M (1991) Structural features in eukaryotic mRNAs that modulate the initiation of translation. J Biol Chem 266:19867-19870

25. Gebauer F, Hentze MW (2004) Molecular mechanisms of translational control. Nat Rev Mol Cell Biol 22:7372-7384. https://doi. org/10.1128/mcb.22.21.7372-7384.2002

26. Komar AA, Hatzoglou M (2011) Cellular IRES-mediated translation. Cell Cycle 10:229-240. https://doi.org/10.4161/cc.10.2. 14472

27. Bergmann C, Zerres K, Rudnik-Schöneborn S et al (2002) Allelic variants in the 5 ' non-coding region of the connexin 32 gene: possible pitfalls in the diagnosis of X linked Charcot-Marie-Tooth neuropathy (CMTX). J Med Genet 39:e58. https://doi.org/10. 1136/JMG.39.9.E58

28. Zuker M (2003) Mfold web server for nucleic acid folding and hybridization prediction. Nucleic Acids Res. https://doi.org/10. 1093/nar/gkg595

29. Hall MP, Unch J, Binkowski BF et al (2012) Engineered luciferase reporter from a deep sea shrimp utilizing a novel imidazopyrazinone substrate. ACS Chem Biol 7:1848-1857. https://doi. org/10.1021/cb3002478

30. Lozano G, Martínez-Salas E (2015) Structural insights into viral IRES-dependent translation mechanisms. Curr Opin Virol $12: 113-120$
31. Martinez-Salas E, Francisco-Velilla R, Fernandez-Chamorro J, Embarek AM (2018) Insights into structural and mechanistic features of viral IRES elements. Front Microbiol 8:2629

32. Johannes G, Carter MS, Eisen MB et al (1999) Identification of eukaryotic mRNAs that are translated at reduced cap binding complex elF4F concentrations using a cDNA microarray. Proc Natl Acad Sci U S A 96:13118-13123. https://doi.org/10.1073/ pnas.96.23.13118

33. Thompson SR (2012) So you want to know if your message has an IRES? Wiley Interdiscip Rev RNA 3:697-705

34. Van Eden ME, Byrd MP, Sherrill KW, Lloyd RE (2004) Demonstrating internal ribosome entry sites in eukaryotic mRNAs using stringent RNA test procedures. RNA 10:720-730. https://doi.org/ 10.1261/rna.5225204

35. Saffran HA, Smiley JR (2009) The XIAP IRES activates 3' cistron expression by inducing production of monocistronic mRNA in the $\beta$ gal/CAT bicistronic reporter system. RNA 15:1980-1985. https://doi.org/10.1261/rna.1557809

36. Southby J, Gooding C, Smith CWJ (1999) Polypyrimidine tract binding protein functions as a repressor to regulate alternative splicing of $\alpha$-actinin mutally exclusive exons. Mol Cell Biol 19:2699-2711. https://doi.org/10.1128/mcb.19.4.2699

37. Lahlou H, Fanjul M, Pradayrol L et al (2005) Restoration of functional gap junctions through internal ribosome entry site-dependent synthesis of endogenous connexins in density-inhibited cancer cells. Mol Cell Biol 25:4034-4045

38. Schiavi A, Hudder A, Werner R (1999) Connexin 43 mRNA contains a functional internal ribosome entry site. FEBS Lett 464:118-122. https://doi.org/10.1016/S0014-5793(99)01699-3

39. Zeitz MJ, Calhoun PJ, James CC et al (2019) Dynamic UTR usage regulates alternative translation to modulate gap junction formation during stress and aging. Cell Rep 27:2737-2747.e5. https:// doi.org/10.1016/j.celrep.2019.04.114

40. López de Quinto S, Martínez-Salas E (1997) Conserved structural motifs located in distal loops of aphthovirus internal ribosome entry site domain 3 are required for internal initiation of translation. J Virol 71:4171-4175

41. Robertson ME, Seamons RA, Belsham GJ (1999) A selection system for functional internal ribosome entry site (IRES) elements: analysis of the requirement for a conserved GNRA tetraloop in the encephalomyocarditis virus IRES. RNA 5:1167-1179. https:// doi.org/10.1017/s1355838299990301

42. Fernandez-Miragall O, Martínez-Salas E (2003) Structural organization of a viral IRES depends on the integrity of the GNRA motif. RNA 9:1333-1344. https://doi.org/10.1261/rna.5950603

43. Bai Y, Wu X, Brennan KM et al (2018) Myelin protein zero mutations and the unfolded protein response in Charcot Marie Tooth disease type 1B. Ann Clin Transl Neurol 5:445-455. https://doi. org/10.1002/acn3.543

44. Taliun D, Harris DN, Kessler MD et al (2021) Sequencing of 53,831 diverse genomes from the NHLBI TOPMed Program. Nature 590:290-299. https://doi.org/10.1038/s41586-021-03205-y

45. Karczewski KJ, Francioli LC, Tiao G et al (2020) The mutational constraint spectrum quantified from variation in 141,456 humans. Nature 581:434-443. https://doi.org/10.1038/s41586-020-2308-7

Publisher's note Springer Nature remains neutral with regard to jurisdictional claims in published maps and institutional affiliations. 